\title{
ON THE EXTENSIONS OF SOME CLASSICAL DISTRIBUTIONS*
}

\author{
by A. SRI RANGA and J. H. MCCABE \\ (Received 13th December 1988)
}

\begin{abstract}
Some properties of polynomials associated with strong distribution functions are given, including conditions for the polynomials to satisfy a three term recurrence relation. Strong distributions that are extensions to the four classical distributions are given as examples.
\end{abstract}

1980 Mathematics subject classification (1985 Revision): 30B70, 33A65

\section{Introduction}

We consider distribution functions whose moments exist for positive and negative values. That is functions $\psi(t)$ which are bounded and non-decreasing in $(-\infty, \infty)$ and for which the moments

$$
\mu_{n}=\int_{-\infty}^{\infty} t^{n} d \psi(t)
$$

are finite for $n=0, \pm 1, \pm 2, \ldots$. Such functions have been described as strong distribution functions because they arise as solutions of strong moment problems (see $[1,2])$. The distribution is called symmetric if all the odd order moments are zero and is called a positive half distribution if all the points of increase are on the positive real axis.

The Hankel determinants are defined by

$$
H_{r}^{(m)}=\left|\begin{array}{cccc}
\mu_{m} & \mu_{m+1} & \cdots & \mu_{m+r-1} \\
\mu_{m+1} & \mu_{m+2} & \cdots & \mu_{m+r} \\
\vdots & \vdots & & \vdots \\
\mu_{m+r-1} & \mu_{m+r} & & \mu_{m+2 r-2}
\end{array}\right| .
$$

for all positive and negative $m$ and $r \geqq 1$, with

*This work was supported by a grant from FAPESP of Brazil. 


$$
H_{-1}^{(m)}=0 \text { and } H_{0}^{(m)}=1 .
$$

For any strong distribution

$$
H_{r}^{(2 m)}>0, \quad r \geqq 0, \quad m=0, \pm 1, \pm 2, \ldots
$$

In the case of a positive half distribution we also have

$$
H_{r}^{(2 m+1)}>0, \quad r \geqq 0, \quad m=0, \pm 1, \pm 2, \ldots,
$$

while for symmetric distributions

$$
H_{2 r+1}^{(2 m+1)}=0 \quad \text { and } \quad(-1)^{r} H_{2 r}^{(2 m+1)}>0, \quad r \geqq 0, \quad m=0, \pm 1, \pm 2, \ldots
$$

The first of these latter results is because the columns of $H_{j}^{(k)}$ are linearly dependent if both $j$ and $k$ are odd. The second follows from the well known Jacobi identity

$$
\left\{H_{r}^{(m)}\right\}^{2}-H_{r}^{(m-1)} H_{r}^{(m-1)}+H_{r+1}^{(m-1)} H_{r-1}^{(m+1)}=0 .
$$

\section{Polynomials related to strong distributions}

Given a strong distribution function $\psi(t)$ we define the polynomials $\left\{Q_{n}(z)\right\}_{0}^{\infty}$ by

$$
\begin{array}{rlrl}
\int_{-\infty}^{\infty} t^{-2[n / 2]+s} Q_{n}(t) d \psi(t) & =0 & & 0 \leqq S \leqq n-1 \\
& =\gamma_{n}, \quad & s=n
\end{array}
$$

for $n \geqq 1$, with $Q_{0}(z)=1$, and $[x]$ denotes integer part of $x$.

In monic form the polynomials can be expressed as

$$
\begin{gathered}
Q_{2 n}(z)=\frac{1}{H_{2 n}^{(-2 n)}}\left|\begin{array}{cccc}
\mu_{-2 n} & \ldots & \mu_{0} \\
\vdots & & \vdots \\
\mu_{-1} & \ldots & \mu_{2 n-1} \\
1 & z & \ldots & z^{2 n}
\end{array}\right| \\
Q_{2 n+1}=\frac{1}{H_{2 n+1}^{(-2 n)}}\left|\begin{array}{llll}
\mu_{-2 n} & \ldots & \mu_{1} \\
\vdots & & \vdots \\
\mu_{0} & \ldots & \mu_{2 n-1} \\
1 & z & \ldots & z^{2 n+1}
\end{array}\right|
\end{gathered}
$$

and, further, 


$$
\gamma_{2 n}=H_{2 n+1}^{(-2 n)} / H_{2 n}^{(-2 n)}, \quad \gamma_{2 n+1}=H_{2 n+2}^{(-2 n)} / H_{2 n+1}^{(-2 n)}
$$

The existence of the polynomials is guaranteed by the positivity of $H_{r}^{(2 m)}, r \geqq 0, m=$ $0, \pm 1, \ldots$, and clearly all $\gamma_{k}$ are positive. It is not difficult to show that the zeros of $Q_{n}(z)$ are real and distinct, for all values of $n \geqq 1$.

A second sequence of polynomials is then defined in the usual way by

$$
P_{n}(z)=\int_{-\infty}^{\infty} \frac{Q_{n}(z)-Q_{n}(t)}{z-t} d \psi(t), \quad n \geqq 0
$$

and clearly $P_{n}(z)$ is a polynomial of degree $n-1$ with leading coefficient $\mu_{0}$.

Strong positive half distributions and strong symmetric distributions belong to those distribution functions for which the following result holds.

Theorem. Let $\psi(t)$ be a strong distribution function such that

$$
H_{2 n}^{(-2 n+1)} \neq 0, \quad n \geqq 0 .
$$

The polynomials $Q_{n}(z)$ and $P_{n}(z)$ each satisfy the three term recurrence relations

$$
\begin{gathered}
R_{2 n}(z)=\left(z-\beta_{2 n}\right) R_{2 n-1}(z)-\alpha_{2 n} R_{2 n-2}(z) \\
R_{2 n+1}(z)=\left\{\left(1+\alpha_{2 n+1}\right) z-\beta_{2 n+1}\right\} R_{2 n}(z)-\alpha_{2 n+1} z^{2} R_{2 n-1}(z)
\end{gathered}
$$

for $n \geqq 1$ with $Q_{0}(z)=1, Q_{1}(z)=z-\mu_{1} / \mu_{0}, P_{0}(z)=0$ and $P_{1}(z)=\mu_{0}$. The coefficients are given $b y$

$$
\begin{gathered}
\alpha_{2 n}=\left\{\frac{H_{2 n}^{(-2 n+1)}}{H_{2 n-1}^{(-2 n+2)}}\right\}^{2} \frac{H_{2 n-2}^{(-2 n+2)}}{H_{2 n}^{(-2 n)}}, \quad \beta_{2 n}=\frac{H_{2 n}^{(-2 n+1)} H_{2 n-1}^{(-2 n+1)}}{H_{2 n-1}^{(-2 n+2)} H_{2 n}^{(-2 n)}} \\
\alpha_{2 n+1}=\frac{H_{2 n+1}^{(-2 n)} H_{2 n-1}^{(-2 n+2)}}{\left\{H_{2 n}^{(-2 n+1)}\right\}^{2}}, \quad \beta_{2 n+1}=\frac{H_{2 n+1}^{(-2 n+1)} H_{2 n}^{(-2 n)}}{H_{2 n+1}^{(-2 n)} H_{2 n}^{(-2 n+1)}}
\end{gathered}
$$

for $n \geqq 1$.

Proof. First for the odd index, write

$$
A(z)=\left\{Q_{2 n+1}(z)-z Q_{2 n}(z)\right\}-\alpha_{2 n+1} z\left\{Q_{2 n}(z)-z Q_{2 n-1}(z)\right\},
$$

a polynomial of degree $2 n$ at most, as

$$
A(z)=-\beta_{2 n+1} Q_{2 n}(z)+B(z),
$$


where $B(z)$ is some polynomial of degree $2 n-1$ at most. Hence from (2.1) it follows that

$$
\int_{-\infty}^{\infty} t^{s} B(t) d \psi(t)= \begin{cases}0, & s=-2 n,-2 n+1, \ldots,-2 \\ -\gamma_{2 n}-\alpha_{2 n+1}\left(\gamma_{2 n}-\gamma_{2 n-1}\right) & s=-1 .\end{cases}
$$

Since $H_{2 n}^{(-2 n)}$ is non zero, then choosing $\alpha_{2 n+1}$ such that

$$
\gamma_{2 n}+\alpha_{2 n+1}\left(\gamma_{2 n}-\gamma_{2 n-1}\right)=0
$$

means that $B(z)$ is identically zero. This gives the required three term relation. Further, as $\gamma_{2 n}$ is positive, choosing $\alpha_{2 n+1}$ in this way is possible only if $\gamma_{2 n}-\gamma_{2 n-1} \neq 0$. Expressing $\gamma_{2 n}$ and $\gamma_{2 n-1}$ in terms of the Hankel determinants and using the Jacobi identity we find that $\gamma_{2 n}-\gamma_{2 n-1} \neq 0$ if $H_{2 n}^{(-2 n+1)} \neq 0$. In this case $\alpha_{2 n+1}$ can be given as in the theorem. With this choice of $\alpha_{2 n+1}$ the value of $\beta_{2 n+1}$ can be found by considering the integral equation

$$
\int_{-\infty}^{\infty} t^{-2 n-1} A(z) d \psi(t)=-\beta_{2 n+1} \int_{-\infty}^{\infty} t^{-2 n-1} Q_{2 n}(z) d \psi(t)
$$

The expression for the even index is verified in a similar fashion by considering

$$
Q_{2 n}(z)-z Q_{2 n-1}(z)=-\beta_{2 n} Q_{2 n-1}(z)-\alpha_{2 n} Q_{2 n-2}(z)+B(z)
$$

where $B(z)$ is some polynomial of degree $2 n-3$ at most.

Having established the recurrence relations for the $Q_{n}(z)$, we then use the definition (2.2) of $P_{n}(z)$ to show that they also satisfy the relations.

The above recurrence relations indicate that the ratios $P_{n}(z) / Q_{n}(z)$ are, for $n=1,2,3, \ldots$, the successive convergents of the continued fraction.

$$
\frac{\mu_{0}}{z-\beta_{1}}-\frac{\alpha_{2}}{z-\beta_{2}}-\frac{\alpha_{3} z^{2}}{\left(1+\alpha_{3}\right) z-\beta_{3}}-\frac{\alpha_{4}}{z-\beta_{4}}-\frac{\alpha_{5} z^{2}}{\left(1+\alpha_{5}\right) z-\beta_{5}}-\frac{\alpha_{6}}{z-\beta_{6}}-\ldots
$$

From the definition of $P_{n}(z)$ we see that

$$
\frac{P_{n}(z)}{Q_{n}(z)}=\int_{-\infty}^{\infty} \frac{1}{z-t} d \psi(t)-\frac{1}{Q_{n}(z)} \int_{-\infty}^{\infty} \frac{Q_{n}(t)}{z-t} d \psi(t)
$$

Expanding the integrand in the second integral in inverse powers of $z$ and using the orthogonality properties of $Q_{n}(z)$ yields 


$$
\frac{P_{2 n}(z)}{Q_{2 n}(z)}=\int_{-\infty}^{\infty} \frac{1}{z-t} d \psi(t)+O\left(\frac{1}{z^{2 n+1}}\right) \quad n \geqq 1
$$

and

$$
\frac{P_{2 n+1}(z)}{Q_{2 n+1}(z)}=\int_{-\infty}^{\infty} \frac{1}{z-t} d \psi(t)+O\left(\frac{1}{z^{2 n+3}}\right) \quad n \geqq 0 .
$$

The symbol $O\left(1 / z^{r}\right)$ denotes a power series in inverse powers of $z$ starting with $1 / z^{r}$.

Since

$$
Q_{2 n}(0)=\frac{H_{2 n}^{(-2 n+1)}}{H_{2 n}^{(-2 n)}}
$$

then under the condition of the above theorem, $Q_{2 n}(0) \neq 0$. On the other hand $Q_{2 n+1}(0)$ may be zero, but, if it is, we can show from the linear system of equations yielded by (2.1) that $Q_{2 n+1}^{\prime}(0) \neq 0$. With these results we can expand the ratio $P_{n}(z) / Q_{n}(z)$ in powers of $z$ and obtain

and

$$
\frac{P_{2 n}(z)}{Q_{2 n}(z)}=\int_{-\infty}^{\infty} \frac{1}{z-t} d \psi(t)+O\left(z^{2 n}\right) \quad n \geqq 1
$$

$$
\frac{P_{2 n+1}(z)}{Q_{2 n+1}(z)}=\int_{-\infty}^{\infty} \frac{1}{z-t} d \psi(t)+O\left(z^{2 n-1}\right) \quad n \geqq 0 .
$$

\section{Examples}

1. The strong Tchebycheff distribution. We first consider the distribution function $\psi_{T}(t)$ given by

$$
\begin{aligned}
d \psi_{T}(t) & =\frac{|t|}{\sqrt{b^{2}-t^{2}} \sqrt{t^{2}-a^{2}}}, & & t \in B \equiv[-b,-a] \cup[a, b] \\
& =0, & & t \notin B
\end{aligned}
$$

with $0<a<b<\infty$.

In the limit as $a \rightarrow 0$ and $b \rightarrow 1$ the distribution becomes the Tchebycheff distribution and so we may view it as an extension to this distribution. Further, since $a>0$, the function has finite moments of negative order and thus we refer to $\psi_{T}(t)$ as a strong Tchebycheff distribution. We have the following result.

Theorem. For the strong Tchebycheff distribution function $\psi_{T}(t)$ defined above the polynomials $Q_{n}(z)$ and $P_{n}(z)$ satisfy the three term recurrence relation (2.3) with $\alpha_{n}$ and $\beta_{n}$ given by 


$$
\begin{array}{lll}
\beta_{n}=0, & \alpha_{2 n}=\gamma, & n \geqq 1, \\
\alpha_{3}=\frac{1}{2} \frac{\lambda^{2}}{\gamma}, & \alpha_{2 n+1}=\frac{1}{4} \frac{\lambda^{2}}{\gamma}, & n \geqq 2,
\end{array}
$$

where $\gamma=a b$ and $\lambda=(b-a)$.

Proof. Consider the continued fraction

$$
\frac{\mu_{0}^{T}}{z}-\frac{a_{2}}{z}-\frac{2 a_{1} z^{2}}{\left(1+2 a_{1}\right) z}-\frac{a_{2}}{z}-\frac{a_{1} z^{2}}{\left(1+a_{1}\right) z}-\frac{a_{2}}{z}-\cdots
$$

in which $a_{2}=\gamma$ and $a_{1}=\lambda^{2} /(4 \gamma)$.

As the coefficients of (3.1) are bounded then the continued fraction coverges uniformly to an analytic function over every bounded closed region in the upper half plane $\operatorname{Im}(z)>0$. See [4, Theorem 9]. Denoting this function by $F(z)$ then

$$
F(z)=\frac{\mu_{0}^{T}}{z}-\frac{a_{2}}{z}-\frac{2 a_{1} z^{2}}{\left(1+2 a_{1}\right) z}-f(z),
$$

where $f(z)$ is a 2-periodic continued fraction which can be written as

$$
f(z)=\frac{a_{2}}{z}-\frac{a_{1} z^{2}}{\left(1+a_{1}\right) z}-f(z) .
$$

Solving for $f(z)$ yields

$$
f(z)=\frac{\left(z^{2}+a_{2}\right) \pm \sqrt{\left(z^{2}+a_{2}\right)^{2}-4 a_{2}\left(1+a_{1}\right) z^{2}}}{2 z} .
$$

If we now choose

$$
a=\sqrt{a_{2}}\left\{\sqrt{1+a_{1}}-\sqrt{a_{1}}\right\},
$$

and

$$
b=\sqrt{a_{2}}\left\{\sqrt{1+a_{1}}+\sqrt{a_{1}}\right\},
$$

then clearly $a_{2}=\gamma$ and $a_{1}=\lambda^{2} /(4 \gamma)$ and we have

$$
f(z)=\frac{1}{2 z}\left\{\left(z^{2}+a b\right) \pm \sqrt{z^{2}-b^{2}} \sqrt{z^{2}-a^{2}}\right\}
$$


The function $f(z)$ has two values but only one of them is appropriate since $F(z)$ must take one value only. We note that $\operatorname{Im} F(z)<0$ whenever $\operatorname{Im}(z)>0$, see [4]. Consequently

$$
f(z)=\frac{1}{2 z}\left\{\left(z^{2}+a b\right)-\sqrt{z^{2}-b^{2}} \sqrt{z^{2}-a^{2}}\right\}
$$

and

$$
F(z)=\mu_{0}^{T} z /\left\{\sqrt{z^{2}-b^{2}} \sqrt{z^{2}-a^{2}}\right\}
$$

The function $F(z)$ can be written alternatively as

$$
F(z)=\frac{\mu_{0}^{T}}{\pi} \int_{B} \frac{1}{z-t} \frac{|t|}{\sqrt{\left(b^{2}-t^{2}\right)} \sqrt{\left(t^{2}-a^{2}\right)}} d t,
$$

a result given in Van Assche [5].

We can show that $\mu_{0}^{T}=\pi$ and hence

$$
F(z)=\int_{-\infty}^{\infty} \frac{1}{z-t} d \psi_{T}(t)
$$

Hence the continued fraction converges to the Stieltjes function of the strong Tchebycheff distribution. Using results given in [3] we can then show that the convergents $P_{n}(z) / Q_{n}(z)$ of (3.1) satisfy (2.4) and (2.5) for this distribution. It is then easy to show that $Q_{n}(z)$ and $P_{n}(z)$ satisfy (2.1) and (2.2) respectively, see [2]. This completes the proof.

We can express $a$ and $b$ in terms of $\gamma$ and $\lambda$,

$$
b=\frac{\gamma}{a}=\lambda+\frac{\gamma}{\lambda}+\frac{\gamma}{\lambda}+\frac{\gamma}{\lambda}+\cdots
$$

Also, by expanding the right hand side of (3.2) we see that the moments of $\psi_{T}(t)$ satisfy

$$
\begin{gathered}
\mu_{2 n}^{T}=\frac{\pi}{4^{n}} \sum_{j=0}^{n} \sigma_{j} \sigma_{n-j}\left(a^{2}\right)^{j}\left(b^{2}\right)^{n-j}, \\
\mu_{-2 n-1}^{T}=\mu_{2 n+1}^{T}=0, \quad \mu_{-2 n-2}^{T}=\mu_{2 n}^{T} /(a b)^{2 n+1},
\end{gathered}
$$

for $n \geqq 0$, where $\sigma_{j}=(2 j) ! /(j !)^{2}$.

2. The strong Legendre distribution. Next we consider 


$$
\begin{aligned}
d \psi_{\mathrm{Le}}(t) & =d t, & & t \in B \equiv[-b,-a] \cup[a, b] \\
& =0, & & t \notin B
\end{aligned}
$$

again with $0<a<b<\infty$.

The moments $\mu_{n}^{L e}, n=0, \pm 1, \pm 2, \ldots$ of this distribution are easily found. As $\psi_{L e}(t)$ is a symmetric distribution function the polynomials $Q_{n}(z)$ and $P_{n}(z)$ each satisfy $(2.3)$. Numerical evidence suggests that

$$
\beta_{n}=0, \quad \alpha_{2 n}=\gamma, \quad \alpha_{2 n+1}=\frac{\lambda^{2}}{\gamma} \cdot \frac{n^{2}}{4 n^{2}-1}, \quad n \geqq 1,
$$

where $\gamma=a b$ and $\lambda=(b-a)$.

The coefficients of the continued fraction

$$
\frac{\mu_{0}^{L e}}{z}-\frac{\alpha_{2}}{z}-\frac{\alpha_{3} z^{2}}{\left(1+\alpha_{3}\right) z}-\frac{\alpha_{4}}{z}-\frac{\alpha_{5} z^{2}}{\left(1-\alpha_{5}\right) z}-\cdots
$$

are bounded and hence the continued fraction converges uniformly over every bounded closed domain in the upper half plane $\operatorname{Im}(z)>0$. (See [4]). Hence, if the values in (3.4) are correct then the continued fraction converges to

$$
\int_{B} \frac{1}{z-t} d \psi_{L e}(t)
$$

In the case when $z=i$ we would then have

$$
\tan ^{-1}\left(\frac{\lambda}{1+\gamma}\right)=\frac{\lambda}{1}+\frac{a_{2}}{1}+\frac{a_{3}}{1}+\frac{a_{4}}{1}+\cdots
$$

where

$$
\begin{gathered}
a_{2 n+2}=\frac{\gamma^{2}\left(4 n^{2}-1\right)}{\left(\lambda^{2}+4 \gamma\right) n^{2}-\gamma}, \quad n \geqq 0 \\
a_{2 n+1}=-\frac{\lambda^{2} n^{2}}{\left(\lambda^{2}+4 \gamma\right) n^{2}-\gamma}, \quad n \geqq 1 .
\end{gathered}
$$

Taking the even contraction leads, after some manipulation, to the well known expansion

$$
\tan ^{-1} x=\frac{x}{1}+\frac{1^{2} x^{2}}{3}+\frac{2^{2} x^{2}}{5}+\frac{3^{2} x^{2}}{7}+\cdots .
$$

A second result in support of (3.4) is the asymptotic behaviour of $\alpha_{n}$. From an analysis similar to that given in Van Assche [5], of the three term recurrence relation (2.3), we find that 


$$
\begin{aligned}
& \sqrt{\alpha_{2 n}}\left\{\sqrt{1+\alpha_{2 n+1}}-\sqrt{\alpha_{2 n+1}}\right\} \rightarrow a \\
& \sqrt{\alpha_{2 n}}\left\{\sqrt{1+\alpha_{2 n+1}}+\sqrt{\alpha_{2 n+1}}\right\} \rightarrow b
\end{aligned}
$$

and clearly the expressions in (3.4) are compatible with these limits.

3. The strong Hermite Distribution. Thirdly we set

$$
d \psi_{H}(t)=e^{-\left(t^{2}+a^{2} / t^{2}\right) / 2} d t-\infty<t<\infty
$$

with $0<a<\infty$. In this case the moments $\mu_{n}^{H}$ satisfy

$$
\begin{gathered}
\mu_{0}^{H}=\sqrt{2 \pi} / e^{a}, \quad \mu_{-2 n-1}^{H}=\mu_{2 n+1}^{H}=0 \\
\mu_{-2 n-2}^{H}=\mu_{2 n}^{H} / a^{2 n+1} \\
\mu_{2 n+2}^{H}=(2 n+1) \mu_{2 n}^{H}+a^{2} \mu_{2 n-2}^{H},
\end{gathered}
$$

for $n \geqq 1$. We can also give $\mu_{2 n}^{H}$ explicitly as

$$
\mu_{2 n}^{H}=\frac{\sqrt{2 \pi}}{2^{3 n} e^{a}} \sum_{r=0}^{n}\left(\begin{array}{l}
2 n+1 \\
2 r+1
\end{array}\right) \sum_{s=0}^{r}\left(\begin{array}{l}
r \\
s
\end{array}\right)(8 a)^{s} \frac{(2 n-2 s) !}{(n-s) !}, \quad n \geqq 0 .
$$

The distribution function $\psi_{H}(t)$ is symmetric and hence the associated polynomials $Q_{n}(z)$ and $P_{n}(z)$ satisfy (2.3). Here computational evidence seems to suggest that

$$
\beta_{n}=0, \quad \alpha_{2 n}=a \quad \text { and } \quad \alpha_{2 n+1}=\frac{n}{a}, \quad n \geqq 1
$$

Again we do not have any analytic proof of this result. However as before, we are able to conjecture that it is true.

If the result is correct then

$$
\int_{-\infty}^{\infty} \frac{1}{z-t} d \psi_{H}(t)=\frac{\mu_{0}^{H}}{z}-\frac{a}{z}-\frac{(1 / a) z^{2}}{(1+1 / a) z}-\frac{a}{z}-\frac{(2 / a) z^{2}}{(1+2 / a) z}-\frac{a}{z}-\cdots .
$$

This continued fraction is uniformly convergent over all bounded closed regions in the half plane $\operatorname{Im}(z)>0$. (See [4, Theorem 9]). Hence, by taking the even part of this continued fraction, we find

$$
\int_{-\infty}^{\infty} \frac{1}{z-t} d \psi_{H}(t)=\frac{\mu_{0}^{H}}{z^{2}-a}-\frac{z^{2}}{z^{2}-a}-\frac{2 z^{2}}{z^{2}-a}-\frac{3 z^{2}}{z^{2}-a}-\cdots .
$$


Then substituting $z /\left(z^{2}-a\right)= \pm i$, we get

$$
1-\sqrt{\pi / 2}\left\{\int_{0}^{\infty} \frac{1}{1+t^{2}} e^{-\frac{1}{2} t^{2}} d t\right\}=\frac{1}{1}+\frac{2}{1}+\frac{3}{1}+\frac{4}{1}+\cdots .
$$

This expansion is correct, and can also be obtained from the $J$-fraction expansion of

$$
\int_{-\infty}^{\infty} e^{-t^{2} / 2} d t /(z-t)
$$

by letting $z=i$.

4. A strong Laguerre distribution. Finally we consider

$$
\begin{aligned}
d \psi_{L a}(t) & =t^{-\frac{1}{2}} e^{-\left(t+a^{2} / t\right) / 2} d t, & & 0<t<\infty \\
& =0, & & -\infty<t \leqq 0
\end{aligned}
$$

with $0<a<\infty$.

This is a positive half distribution and hence the polynomials $Q_{n}(z)$ and $P_{n}(z)$ do satisfy (2.3). It appears that

$$
\begin{array}{ll}
\beta_{2 n-1}=2 n-1+a, & \beta_{2 n}=a, \\
\alpha_{2 n-1}=(2 n-2) / a, & \alpha_{2 n}=(2 n-1) a
\end{array}
$$

for $n \geqq 1$.

These results essentially follow from the strong Hermite case. Substituting $z^{2}=z$ and $t^{2}=u$ in (3.5) yields an $M$-fraction expansion for

$$
\int_{0}^{\infty} t^{-1 / 2} e^{-1 / 2\left(t+a^{2} / s\right)} d t /(z-1)
$$

The coefficients of this $M$ fraction can then be used to derive the $\alpha_{j}$ and $\beta_{j}$.

The moments $\mu_{n}^{L a}$ of this distribution function satisfy

$$
\mu_{n}^{L a}=\mu_{2 n}^{H}
$$

for all positive and negative $n$.

\section{REFERENCES}

1. W. B. Jones, O. Niastad and W. J. Thron, Laurent Polynomials and the strong Hamburger moment problem, J. Math. Anal. Appl. 98 (1984), 528-554. 
2. W. B. Jones, W. J. Thron and H. WaAdeland, A strong Stieltjes moment problem, Trans. Amer. Math. Soc. 261 (1980), 503-528.

3. A. SRI RANGA, J-fractions and the strong moment problems, in Analytic Theory of Continued Fractions (Proceedings (W. J. Thron, Ed), Pitlochry, Scotland, 1985; Lectures Notes in Mathematics, 1199, Springer-Verlag, 1986).

4. A. SRI Ranga, Convergence properties of a class of J-fractions, J. Comput. Appl. Math. 19 (1987), 331-342.

5. W. VAN Assche, Asymptotic properties of orthogonal polynomials from their recurrence formula, I, J. Approx. Theory 44 (1985), 258-276.

ICMSC-University of Sao Paulo

Sao Carlos, SP

Brazil
Department of Mathematical Sciences UNIVERSITY OF $\mathrm{Sr}$ ANDREWS

St ANDREws, Fife

SCOTLAND 